\title{
A SDN Based Scalable Layer 2 Network for Next Generation Internet Testbed
}

\author{
Yanwei $\mathrm{Xu}$ \\ Shanghai Engineering Research Center for Broadband \\ Networks and Applications \\ Shanghai, China \\ ywxu@bnc.org.cn
}

\author{
Xiaoyuan Lu \\ Shanghai Engineering Research Center for Broadband \\ Networks and Applications \\ Shanghai, China \\ xylu@bnc.org.cn
}

\author{
Tairan Zhang \\ Shanghai Engineering Research Center for Broadband Networks and Applications \\ Shanghai, China \\ trzhang@bnc.org.cn
}

\begin{abstract}
The demands for the next generation internet testbeds are becoming more and more intense recently. However, there are three problems need to solve, i.e., the ever-increasing number of actual or virtual machines demands, effectively manage these different machines and maintain the effectiveness. A scalable layer 2 network that across the whole internet is a very important technology to solve them. This paper proposes a new software defined network (SDN) based scalable layer 2 network framework and its significant characteristic is to build a scalable layer 2 network that across the whole internet by using SDN's technologies. Our framework has high performances because: 1) The operation of encapsulating and unpacking packets is not necessary; 2) Routing paths can be pre-computed; 3) Traffic engineering to be implemented. And then the proposed framework has good scalability because its addressing resolution can be done either by a centralized query or by transmitting a broadcast packet in the internet. And the two ways can be swapped flexibly by the load balancing.
\end{abstract}

Keywords-Testbeds, SDN, OpenFlow, Scalable Layer 2

\section{INTRODUCTION}

Testbed [5] is a test development environment that can simulate the actual environment of a large number of tests. It has showed great potential on many applications through the composition of technology tests and applications. In the past few years, small-scale testbeds have been presented to reduce the costs of hardware in the majority of cases, and they have been concerned by many researchers for evaluating the quality of their solutions, but they tend to only offer limited support in terms of heterogeneity, scalability and mobility in many complicated applications that owns tightly coupled network and software architecture.

In order to overcome the limitations in scale, a number of large-scale testbeds have been developed in the recent years $[7,8,11,14]$. These testbeds are built with future complicated network equipments and computers used in real large-scale environments, and they are becoming a hot research topic as next generation Internet testbeds. These testbeds often consist of a few dozen actual or virtual machines. Although this explicit need for large-scale testbeds, three critical questions are posed:

- How do we deal with the ever-increasing number of actual or virtual machines demands in large-scale testbeds?

- How do we provide a common virtual management platform for different large-scale testbeds that own different machines?

- How can we maintain the effectiveness of large-scale testbeds?

A scalable layer 2 network that may across the entire Internet will be an effective answer to these problems. Scalable layer 2 network architecture is derived from the need for data center service requirements. And it introduces the flexibility for allowing the insertion or movement of a device in a transparent fashion from the perspective of the IP layer. Virtualization technologies not only increase the density of managed virtual servers in the data center, but also exacerbating the need for scalable layer 2 network.

Software defined network (SDN) $[6,10]$ may be another effective answer to solve large-scale testbeds' problems. SDN is an emerging paradigm in computer networking that allows a logically centralized software program to control the behavior of an entire network. But a pure SDN technology is still difficult to solve those problems mentioned above, the reasons are: (1) the configurations of controller in a pure SDN is very high, thus it is difficult to provide excellent performance for large-scale testbeds; (2) its scalability will become very poor due to the restrictions of flow table entries.

In order to integrate their respective advantages of scalable layer 2 network and SDN. This paper proposes a new SDN based scalable layer 2 network framework, which has three functional modules: fabric construction, broadcast tree creation and traffic engineering. 
This paper is organized as follows: related works are described in section 2. In section 3, The proposed framework and problem definition are presented. Finally, concluding remarks are given in section 4 .

\section{RELATED WORK}

Software-defined networking (SDN) is an approach to computer networking which evolved from work done at UC Berkeley and Stanford University around 2008 [10]. SDN allows network administrators to manage network services through abstraction of lower level functionality. This is done by decoupling the system that makes decisions about where traffic is sent (the control plane) from the underlying systems that forwards traffic to the selected destination (the data plane). The inventors and vendors of these systems claim that this simplifies networking [6]. When a packet arrives at a switch in a conventional network, rules built into the switch's proprietary firmware tell the switch where to forward the packet. The switch sends every packet going to the same destination along the same path - and treats all the packets the exact same way.

SDN requires some methods for the control plane to communicate with the data plane. One such mechanism, OpenFlow, is often misunderstood to be equivalent to SDN, but other mechanisms could also fit into the concept. The Open Networking Foundation was founded to promote SDN and Open-Flow as marketing using the term cloud computing became popular. SDN has attracted lots of efforts from both the research and industry community $[4,9,13]$ after it was proposed.

In the existing SDN studies such as [9], the Controller is responsible for all the communications in the network: processing every packet-in messages and calculating the routing pathes for each time of data transmission. This operation manner brings an extremely heavy burden onto the Controller, which highly challenges its efficiency and limits the scalability of SDN in practical networks [12]. This paper employs a totally different operation style for the Controller which highly releases its workload and solves the scalability problem:

- The routing pathes of each pair of switches in the network are pre-computed at the booting up stage of the network;

- The ARP requesting messages can be resolved both by the Controller and the broadcasting in the networks, in a load balancing manner.

\section{Problem Definition AND the Framework}

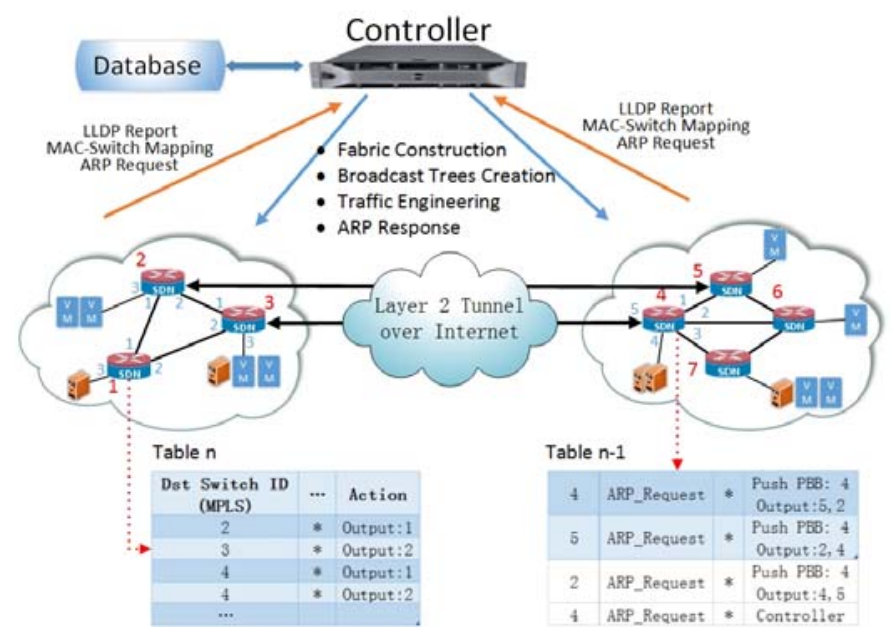

Fig. 1. Framework of the Proposed Internet Network Testbed

AS shown in Fig.1, our International Network Testbed is constituted by a number of sub-networks. Each sub-network contains a set of OpenFlow switches, each of which is linked with some hosts (physical machines or virtual machines (VM)). Some pairs of switches of different sub-networks have established layer 2 network tunnels (such as the GRE tunnel for connecting sub-networks to form a large-scale layer 2 network. The core of the testbed is a SDN Controller, which organizes the sub-networks to be a single fabric.

In Fig.1, the testbed has two sub-networks, where the red numbers indicate the switch IDs and the blue ones are the switch port numbers of each link. There are two tunnels between the two pairs of switches of the two sub-networks.

The main characteristic of the proposed method are as follows:

- All the switches use the LLDP protocol to find their neighbourhoods, the link attributes and the attached hosts, and then report them to the Controller. Hence, the Controller has the full topology of the whole network, which are used for fabric construction and broadcast tree creation at the network booting up stage.

- In each time of unicasting, the ARP requesting packet can be processed by the Controller and the broadcasting in the networks, in a load balancing manner. The ARP responsing message not only contains the MAC address of the destination host, but also the access switch ID of the destination host. The queried switch ID is used to tag the data packets by the access switch of the source host, and then the packets are routed using the destination switch ID by the flow table entries created in the booting up stage. 


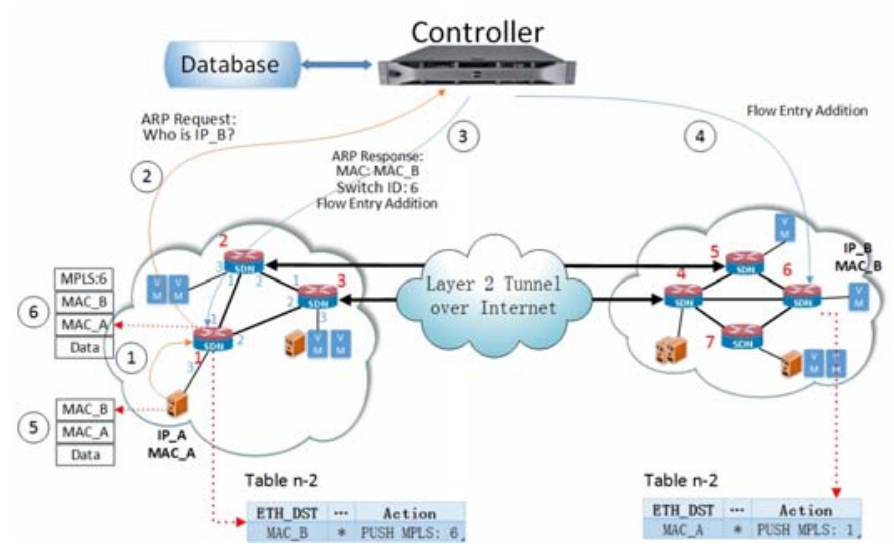

Fig. 2. Unicasting Process

Figure 2 depicts the process of a unicasting. When a host $A$ wants communicate with a host $B$ at the first time, it send an ARP request message to its access switch. If $B$ is directly connected to A's access switch, the ARP request is resolved in the traditional manner; otherwise, the switch will forward the ARP request message to the Controller or the other switches, determined by the corresponding priorities of the flow entries.

If the Controller received the ARP request message, it will search the MAC address of $B$ and the ID of its access switch in its database that storing the topology. And then, the Controller sends an ARP responding message to $A$ and two OpenFlow messages to the two access switches of $A$ and $B$ (switch 1 and 6) for loading the two flow entries as shown in the bottom of Fig.2, whose purposes are to tag the data packets using the destination switch IDs. Note that such flow entries must be stored in the flow table prior to the table for storing the entries that are created in the booting up stage.

After the ARP resolving is finished, the data packets will be tagged by the destination switch by the access switch of the source host. And then, the data packets are forwarded in the switch overlay based on the destination switch ID.

\section{EXPERIMENTS}

We build a testbed running on the OVS [3] based platform to evaluate the efficiency of SFabric. And the experimental results are mainly focused on the average latencies of establishing a communicating path between two hosts and the end-to-end round-trip time of transmitting a packet.

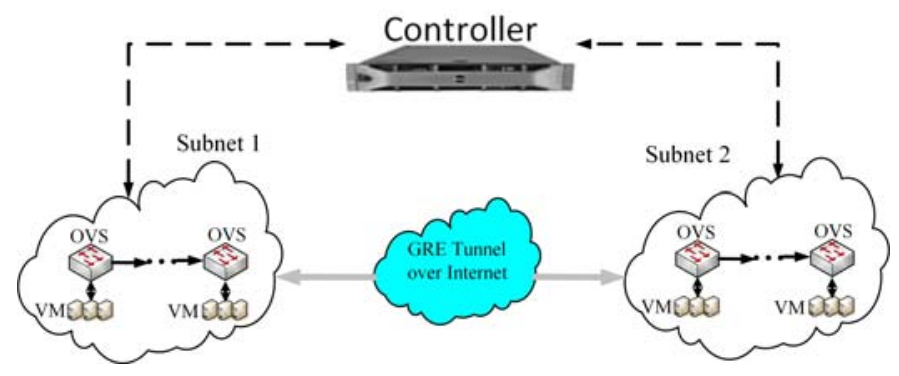

Fig. 3. Testbed of the Experiments
In the experiments, two merits are used to validate the efficiency of the proposed method:

- PCT (Path Construction Time): the time needed for constructing the communicating paths for two hosts.

- $\quad$ RTT (End-to-End Round-Trip Time): the time used for transmitting a packet between two hosts.

Because the number of switches along a path will affect the values of PCT and RTT, for simply focusing on the results of PCT and RTT but without generality, the topology of the testbed is linear, as shown in Fig.3. The testbed is composed of two subnets of two universities in Shanghai, which are connected by the GRE layer 2 tunnel across the public Internet. Each subnet contains at most 100 OVS bridges created by Mininet [1] using the linear topology. The GRE tunnel is created in the two right-most and left-most switches of the two subnets. Each subnet is running on a PC server (Lenovo Think Server R630, CPU is Intel Xeon E5-2630*2, RAM is $16 \mathrm{G}$ DDR3-1333). We implement the proposed method based on the OpenDayLight [2] platform. And in the experiments, two other technologies are compared:

- STP: the traditional spanning-tree protocols.

- ODL: the build-in L2 switching of OpenDayLight, which works as the most existing SDN studies that the Controller have to install OpenFlow table entries at every switch along the path between two communicating hosts.

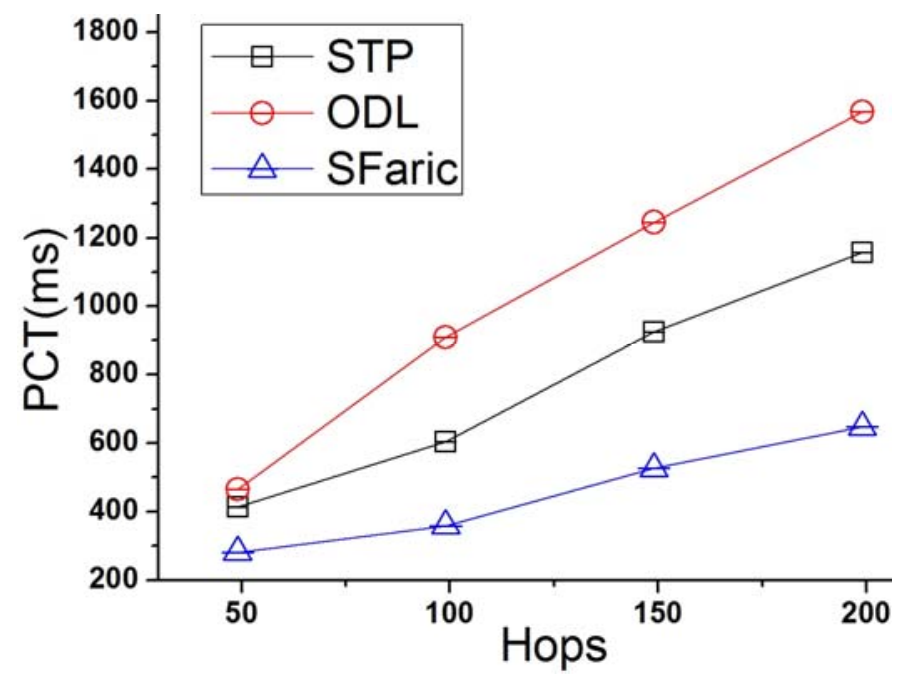

Fig. 4. The comparisons of the average PCTs among STP, ODL and SFabric with different hops

The PCTs, as shown in Fig.4, are obtained while five VMs of the first bridge (the leftmost) are communicating with other VMs connecting to the bridges which are 49, 99, 149 and 199 hops away, respectively. Since SFabric has finished the fabric construction and made every switch know the routes to other switches after network booting up, the Controller just needs to install two flow table entries into the first and last switches while creating a path for a pair of hosts. Compared with other existing OpenFlow solutions that should install the 
flow table entry into every switch along that path, the path construction time (PCT), mainly spent on ARP resolving and the process of installing the flow table entries, are highly reduced. The PCT of ODL is the longest since it should install the flow table entry into every switch along a path, which is very time-consuming. The PCTs of STP are in the middle due to its broadcasting manner in ARP resolving. Lastly, SFabric just needs to install two flow entries into the first and last switches of a path, so its PCT is the shortest.

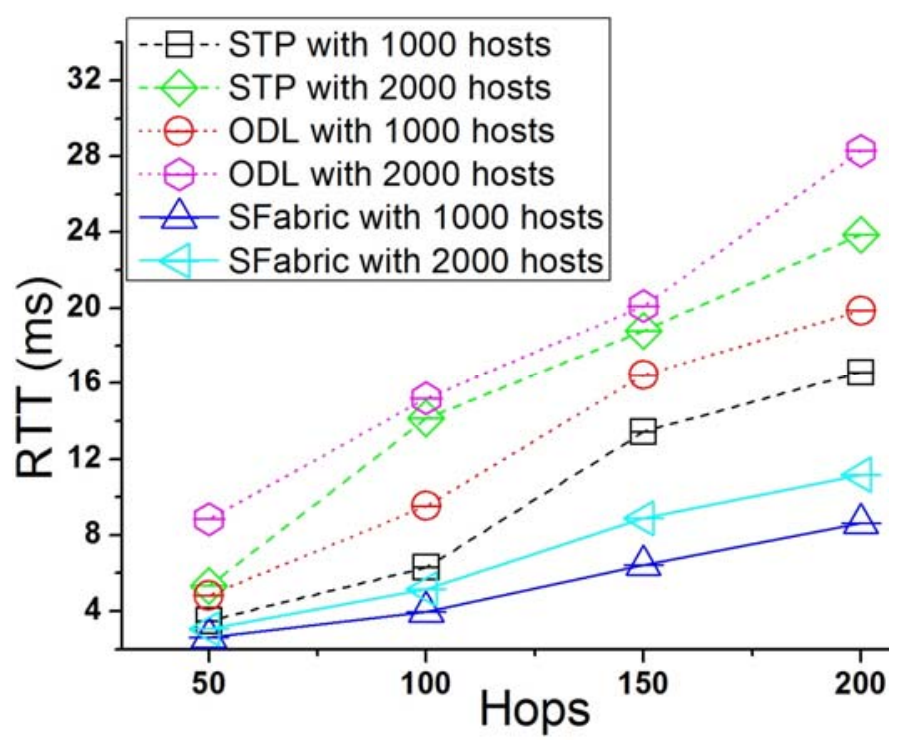

Fig. 5. The comparisons of RTTs among STP, ODL and SFabric with different numbers of hosts

Since the amount of hosts in a network will affect the RTT, we set a scenario where various numbers of hosts exist in a certain subnetwork (i.e., SFabric with 1000 hosts in Fig.5 means there are 1000 hosts exist between the 1st OVS and another OVS when SFabric is used) in order to observe the disparities among the RTTs of STP, ODL and SFabric. From Fig. 5 we can see that the RTTs of SFabric with different hosts are very close whereas the RTTs of ODL are much different from each other, which prove the delay influence caused by the number of hosts is limited to SFabric but serious to ODL. This is because the reduction of flow table entries since the time used for finding out the right output at each switch is much less than ODL. Furthermore, as the hosts or switches increase, the gaps among the RTTs of those three solutions are also enlarged correspondingly.

\section{CONCLUSION}

In this paper, we propose a large layer 2 network based on SDN technologies for constructing an Internet Testbed. A central controller is used to manage the routing maps among the switches. But different from other SDN solutions, it employs a totally different operation style for the Controller, which highly releases its workload, forwards flows more efficiently and solves the scalability problem.
In the future, we will test our framework in the real network and promote its performance. And the traffic engineering components will be developed and studied in the future. Further more, the ARP resolving is only done by the Controller, which will pose a heavy burden to the Controller. How to relieve such burdens would be another future study direction.

\section{ACKNOWLEDGMENT}

We would like to thank Lingwei Chu (Shanghai Engineering Research Center for Broadband Networks and Applications) for his support from the research on the SDN system with high reliability based on open architecture (13511500400). Moreover, we also appreciate the help from the shanghai SDN industrial technology and innovation strategic alliance (13DZ0510500).

\section{REFERENCES}

[1] Mininet. http://mininet.org.

[2] OpenDayLight.http://OpenDayLight.org.

[3] OpenVSwitch. http://openvswitch.org.

[4] Programmable flow networking. http://www.necam.com/SDN/.

[5] T.Baumgartner, I.Chatzigiannakis, M.Danckwardt, C.Koninis, A.Kroller G.Mylonas, D.Pfisterer, and B.Porter. Virtualising testbeds to support large-scale reconfigurable experimental facilities.In Wireless Sensor Networks, EWSN 2010. Proceedings, pages 210--223, 2010.

[6] O.N. Foundation. Software-Defined Networking: The New Norm for Networks. White paper. 2013.

[7] M.Haque, K.Pawlikowski, and S.K. Ray. Challenges to development of multipurpose global federated testbed for future internet experimentation. In The 9th IEEE/ACS International Conference on Computer Systems and Applications, AICCSA 2011, Sharm El-Sheikh, Egypt, December 27-30, 2011, pages 289--292, 2011.

[8] J.Hu, C.Yang, and T.Liu. Design and implementation of an efficient and programmable future internet testbed in taiwan. Comput. Sci. Inf. Syst., 10(2):825--842, 2013.

[9] S.Jain, A.Kumar, S.Mandal, J.Ong, L.Poutievski, A.Singh, S.Venkata, J.Wanderer, J.Zhou, M.Zhu, J.Zolla, U.Holzle, S.Stuart, and A.Vahdat. B4: experience with a globally-deployed software defined wan. In ACM SIGCOMM 2013 Conference, SIGCOMM'13, Hong Kong, China, August 12-16, 2013, pages 3--14, 2013.

[10] N.McKeown, T.Anderson, H.Balakrishnan, G.M. Parulkar, L.L. Peterson, J.Rexford, S.Shenker, and J.S. Turner. Openflow: enabling innovation in campus networks. Computer Communication Review, 38(2):69--74, 2008.

[11] I.M. Moraes, D.M.F. Mattos, L.H.G. Ferraz, M.E.M. Campista, M.G.Rubinstein, L.H. M.K. Costa, M.D. deAmorim, P.B. Velloso, O.C. M.B. Duarte, and G.Pujolle. FITS: A flexible virtual network testbed architecture. Computer Networks, 63:221--237, 2014.

[12] A.Voellmy and J.Wang. Scalable software defined network controllers. In ACM SIGCOMM 2012 Conference, SIGCOMM'12, Helsinki, Finland - August 13 - 17, 2012, pages 289--290, 2012.

[13] A.Voellmy, J.Wang, Y.R. Yang, B.Ford, and P.Hudak. Maple: simplifying SDN programming using algorithmic policies. In ACM SIGCOMM 2013 Conference, pages 87--98, 2013.

[14] Y.Zhuang, L.Law, A.Rafetseder, L.Wang, I.Beschastnikh, and J.Cappos. Sensibility testbed: An internet-wide cloud platform for programmable exploration of mobile devices. In 2014 Proceedings IEEE INFOCOM Workshops, Toronto, ON, Canada, April 27 - May 2, 2014, pages 139-$140,2014$. 